\title{
Ovarian stimulation in patients with breast cancer
}

\author{
Elkin Muñoz ${ }^{1}$, Naira González¹, Luis Muñoz², Jesús Aguilar ${ }^{1}$ and Juan A García Velasco ${ }^{3}$ \\ ${ }^{1}$ IVI Vigo, Plaza Francisco Fernández del Riego, 7, Vigo 36203, Pontevedra, Spain \\ ${ }^{2}$ Fundación Universitaria de Ciencias de la Salud, Bogotá, Cundinamarca 11001000, Colombia \\ 3IVI Madrid, Universidad Rey Juan Carlos, Fuenlabrada, Madrid 28023, Spain
}

Correspondence to: Jesús Aguilar Prieto. Email: Jesus.Aguilar@ivi.es

\begin{abstract}
Breast cancer is the most prevalent malignancy among women under 50. Improvements in diagnosis and treatment have yielded an important decrease in mortality in the last 20 years. In many cases, chemotherapy and radiotherapy develop side effects on the reproductive function. Therefore, before the anti-cancer treatment impairs fertility, clinicians should offer some techniques for fertility preservation for women planning motherhood in the future.

In order to obtain more available oocytes for IVF, the ovary must be stimulated. New protocols which prevent exposure to increased estrogen during gonadotropin stimulation, measurements to avoid the delay in starting anti-cancer treatment or the outcome of ovarian stimulation have been addressed in this review.

There is no evidence of association between ovarian stimulation and breast cancer. It seems that there are more relevant other confluent factors than ovarian stimulation. Factors that can modify the risk of breast cancer include: parity, age at full-term birth, age of menarche,
\end{abstract} and family history.

There is an association between breast cancer and exogenous estrogen. Therefore, specific protocols to stimulate patients with breast cancer include anti-estrogen agents such as letrozole. By using letrozole plus recombinant follicular stimulating hormone, patients develop a multifollicular growth with only a mild increase in estradiol serum levels.

Controlled ovarian stimulation (COS) takes around 10 days, and we discuss new strategies to start COS as soon as possible. Protocols starting during the luteal phase or after inducing the menses currently prevent a delay in starting ovarian stimulation.

Patients with breast cancer have a poorer response to COS compared with patients without cancer who are stimulated with conventional protocols of gonadotropins.

Published: 03/02/2015

Received: 20/08/2014

ecancer 2015, 9:504 DOI: 10.3332/ecancer.2015.504

Copyright: (c) the authors; licensee ecancermedicalscience. This is an Open Access article distributed under the terms of the Creative Commons Attribution License (http://creativecommons.org/licenses/by/3.0), which permits unrestricted use, distribution, and reproduction in any medium, provided the original work is properly cited. 
Although many centres offer fertility preservation and many patients undergo ovarian stimulation, there are not enough studies to evaluate the recurrence, breast cancer-free interval or mortality rates in these women.

Keywords: breast cancer, controlled ovarian stimulation, fertility preservation, letrozol

\section{Introduction}

Breast cancer is the most common neoplasia among women under 50. It is estimated that during 2012, 1.67 million new cases of breast cancer were diagnosed worldwide; $25 \%$ of all cancers, according to WHO data. This percentage is somewhat higher in industrialized countries [1, 2].

Breast cancer is the most prevalent neoplasia type in women of reproductive age. In Europe, the incidence of breast cancer in premenopausal women is $30 / 100,000[3,4]$. While in the United States it is estimated that one in every 202 women will develop an invasive breast cancer before the age of 39 [2].

Progress made in the early diagnosis and treatment enables an increasingly higher number of women to overcome breast cancer. In 2009 , the risk of death from cancer, of any type, in the United States was estimated at 20\% lower than in 1991 [3]. The reduction in mortality makes patients look forward to fulfill other objectives once they overcome the disease. Fertility is one of those objectives which concerns patients who survive cancer.

Cancer treatments affect reproduction in women. The adverse effect that chemotherapy and radiation therapy have on fertility is well known. Initial studies demonstrated follicular depletion and the functional alteration of the ovary $[5,6]$, although the result is not always ovarian failure. In some cases, treatments are associated with the recovery of menstrual cycles, but with reproductive problems during the long-term. Thus, the implementation of in vitro fertilization and embryo cryo-preservation shortly after the treatment may involve some possible genetic risks for the oocytes in development [7]. Breast cancer is the most frequent cause for preserving fertility. It is estimated that approximately half of the women undergoing treatment for fertility preservation are patients with breast cancer [8].

\section{Chemotherapy in breast cancer}

There is evidence that chemotherapy produces an increase in disease-free survival and overall survival of patients with breast cancer. The goal of chemotherapy is to eradicate the micro-metastatic disease responsible for the recurrence and progression of the disease $[9,10]$.

The administration of six cycles of anthracycline-based chemotherapy reduces the risk of annual death by $38 \%$ for women younger than 50 , and approximately by $20 \%$ for women between 50 and 69 years old. The benefit is independent of the use of tamoxifen, the status of the estrogen receptor, and the nodal status or other characteristics of the tumour. These diagrams, based on anthracyclines, are clearly more effective than other protocol type CMF (cyclophosphamide, methotrexate and fluorouracil) $[9,10]$. The addition of taxanes combined with anthracyclines has demonstrated superiority in the survival rate for tumours which are biologically more aggressive [10].

The impact of the treatments for ovarian function depends on the chemotherapeutic agents used, the dose, the age, ovarian reserve, and the association of several drugs or radiotherapy [11, 12].

Patients with stage II breast cancer and those with stage I tumours larger than $1 \mathrm{~cm}$ should be warned about the effect of gonad-toxic chemotherapy, since this will form a part of their therapeutic routine [13]. The risk of amenorrhea is not equal for all types of treatments but it varies in relation to the type of chemotherapy and radiotherapy (Table 1).

The addition of taxanes to the treatment schemes based on anthracyclines (AC, FEC, and FAC) is a recommendation of randomized clinical trials [14]. The gonadal toxic effect of taxanes and trastuzumab is not well established, and both are essential drugs in the adjuvant treatment of breast cancer. The addition of taxanes to schema AC (cyclophosphamide, doxorubicin) produces a significant increase in the rate of amenorrhea post-chemotherapy in women over 40, but not in those who are younger [15]. 
Table 1. Risk of permanent amenorrhea in women treated with chemotherapy and radiotherapy.

\begin{tabular}{|c|c|}
\hline High Risk $>80 \%$ & $\begin{array}{l}\text { External radiotherapy that includes the pelvic region } \\
\text { CMF, CEF, CAF x } 6 \text { cycles. Women }>40 \text { years old } \\
\text { (CMF: cyclophosphamide, methotrexate and fluorouracil } \\
\text { CEF: cyclophosphamide, epirubicin, fluorouracil } \\
\text { CAF: cyclophosphamide, doxorubicin, fluorouracil) }\end{array}$ \\
\hline Intermediate Risk & $\begin{array}{l}\text { CMF, CEF, CAF x } 6 \text { cycles. Women } 30-39 \text { years old } \\
\text { AC } \times 4 \text { in women }>40 \text { years } \\
\text { (Doxorubicin/cyclophosphamide) }\end{array}$ \\
\hline Low Risk $<20 \%$ & $\begin{array}{l}\text { CMF, CEF, CAF } \times 6 \text { cycles in women }<30 \text { years old } \\
A C \times 4 \text { in women }<40 \text { years old }\end{array}$ \\
\hline Very Low Risk or no risk & $\begin{array}{l}\text { Vincristine } \\
\text { Methotrexate } \\
\text { Fluorouracil }\end{array}$ \\
\hline Unknown Risk & $\begin{array}{l}\text { Taxanes } \\
\text { Oxaliplatin } \\
\text { Irinotecan } \\
\text { Monoclonal Antibodies (trastuzumab, bevacizumab and } \\
\text { cetuximab) } \\
\text { Tyrosine-Kinase Inhibitors (ertolinib, imatinib) }\end{array}$ \\
\hline
\end{tabular}

Table is based on ASCO recommendations on fertility preservation in cancer patients [11]

\section{Association between ovarian stimulation and cancer}

There is no absolute evidence of association between ovarian stimulation for IVF and cancer [16]. According to a recent meta-analysis, it has not been possible to associate ovarian stimulation in IVF treatments with the appearance of ovarian, endometrial, or cervical cancer, although the longest period of follow-up in the studies was 10 years [17]. A retrospective cohort study that included more than 20,000 women, with a mean follow-up of 15 years, found a greater number of borderline ovarian tumours in patients undergoing IVF compared with sub-fertile women who did not undergo IVF. This difference was not found for epithelial ovarian cancer [18].

The studies which are looking for the relationship between breast cancer and ovarian stimulation show conflicting results. They are case and control studies with an insufficient number of patients [19, 20]. It seems logical to think that if there were any association between controlled ovarian stimulation (COS) and breast cancer, the tumours would develop after stimulating a woman who should present an over expression of oestrogen receptors (ER). One study examines the histological and molecular characteristics of breast cancers diagnosed until 24 months after COS. When compared with a control group of similar age without exposure to COS, no differences between the two groups were found [21].

\section{How to stimulate a patient with breast cancer?}

There are several options available for preserving fertility in cancer patients: embryo cryo-preservation, vitrification of oocytes, ovarian tissue freezing, and maturation of oocytes in vitro. These last two techniques, which are less widely used, are considered experimental despite there being protracted experience with its use [22-24]. The cryo-preservation of embryos is the most widespread method being used and in which there is a greater experience. In recent years, vitrification of oocytes has become a widely used technique [25]. Cryo-preservation of embryos, such as oocytes, is usually carried out after COS. 
A conventional IVF cycle requires approximately 10-14 days of COS with gonadotropins to achieve multi-folicular development. This results in an elevation of the serum estradiol, up to 10-15 times higher than physiological levels. To avoid this elevation of estradiol in patients with breast cancer, many protocols have been developed for stimulation with gonadotropins associated with tamoxifen or Aromatase inhibitors (letrozole) [26-32]. The peak of estradiol in the protocols of ovarian stimulation with tamoxifen or with letrozole is slightly higher than the levels achieved in a natural cycle; around 300-350 pg/ml [27].

The most widely used protocol to stimulate patients with breast cancer is the oral administration of letrozole $5 \mathrm{mg}$ from day $2-3$ of the cycle, in conditions of ovarian quiescence [follicular stimulating hormone (FSH) $<13$ IU/L/E2 < 60 pg/ml]. After 2 days of treatment with letrozole, a variable dose of recombinant $\mathrm{FSH}(\mathrm{rFSH})$ between 150 and $300 \mathrm{IU} /$ day is added. When the concentration of serum estradiol exceeds $250 \mathrm{pg} / \mathrm{ml}$ or the follicles reach a size greater than $13 \mathrm{~mm}$ in diameter, administration of GnRH antagonists is started to avoid the premature peak of LH. Follicular growth is monitored until at least two of the follicles reach $20 \mathrm{~mm}$ in diameter and at that moment ovulation is triggered with the agonists of $\mathrm{GnRH}$. By comparing the use of $\mathrm{GnRH}$ agonists versus hCG trigger ovulation, it was found that the agonists achieved a greater and faster decline of the estradiol levels without reducing the number of mature oocytes collected or the fertilization rate [28, 30].

This protocol with letrozole, along with final $\mathrm{rFSH}$ and the induction of ovulation with $\mathrm{GnRH}$ agonists (triptorelin), has been implemented in an extended form, independent of the molecular phenotype of breast cancer [26-32].

There is no evidence that the exposure of the oocytes to letrozole increases congenital defects at birth. However, the effect is not known if the embryos are exposed to systemic letrozole [31, 32].

Novartis, the company responsible for the production of Femara (letrozole) does not recommend the use of this drug as an inducer of ovulation. Novartis states that the drug should not be used in women who may become pregnant, during pregnancy and/or breast-feeding, because there is a potential risk of harm to mother and fetus, including risk of fetal malformations. A study of 911 newborns shows similar overall rates of major and minor congenital malformations among newborns from mothers who conceived after letrozole or clomiphene citrate [33]. In Spain, the Ministry of Health, Social Services, and Equality has approved its compassionate use in patients with breast cancer.

\section{How to avoid a delay in the start of the stimulation?}

In oncology, patients are not advised to delay the start of COS. It is therefore usual to begin the stimulation outside days 2-3 of the menstrual cycle. The strategy varies according to the time of the cycle.

In the early proliferative phase, when there is still no dominance follicular, stimulation starts even if the patient is not on day $2-3$ of the cycle. If the cycle is in a proliferative phase after the follicular dominance and the follicular size is $\geq 18 \mathrm{~mm}$ in average diameter, the puncture of the follicle and the vitrification of the oocyte are performed. Subsequently, the GnRH antagonist is applied for 5 days. If the dominant follicle is less than $18 \mathrm{~mm}$, it is stimulated with minimal doses of $\mathrm{FSH}$, until the follicle reaches $18 \mathrm{~mm}$ in diameter and follicular puncture is performed. The $\mathrm{GnRH}$ antagonist is then administered for 5 days.

When the patient is in the secretory phase of the cycle, the ovulation is confirmed by ultrasound along with the analytical determination of estradiol and progesterone. The $\mathrm{GnRH}$ antagonist is administered for 4-5 days, and then stimulation is begun. The objective of the antagonists of $\mathrm{GnRH}$ is to achieve estradiol levels below $60 \mathrm{pg} / \mathrm{ml}$ and not to delay treatment until the start of a new physiological cycle [28, 30, 34, 35].

In some cases, the administration of gonadotropins had been attempted during the secretory phase to simulate the appearance of a second wave of endogenous gonadotropins that occur in a physiological way. However, ovarian stimulation which is started during the secretory phase is a strategy which has variable results [35]. 


\section{Responses to ovarian stimulation in cancer patients}

Cancer is associated with increased states of catabolism and malnutrition. Many patients experience weight loss, which could affect the hypothalamic-pituitary axis and lead to a reduction in reproductive capacity. The occurrence of psychological stress could lead to an increase in prolactin and endogen opioids. Further to this, levels of gonadotropins could decrease, thus affecting fertility [36]. Therefore, illness could affect the ovary and its responsiveness, even prior to chemotherapy and radiotherapy [37, 38].

Studies of ovarian reserves in those whose Anti-Mullerian Hormone (AMH) levels have been measured show that women with cancer have lower levels of AMH prior to receiving chemotherapy than the expected values according to their age group [39]. The antral follicle count is also lower in women with cancer compared to a healthy age-matched control group [40]. Recently, some studies point to the possible association of BRCA1 mutation with the reduced ovarian response to stimulation [41].

According to these considerations, various different studies have compared the result of COS in cancer patients and cancer-free IVF patients. A recent meta-analysis concludes that women with cancer who undergo COS in order to maintain fertility during cancer treatment produce fewer oocytes than healthy patients of similar age [38]. Other findings include a longer duration of COS and higher levels of gonadotropins in cancer patients [34].

Our group has demonstrated that women with breast cancer produce 2.4 fewer oocytes than women of a similar age stimulated via a conventional IVF protocol [36]. No prospective randomized studies have currently taken place which compare clomiphene citrate or tamoxifen and gonadotropins with a conventional protocol consisting only of gonadotropins [40].

Other groups produced different results. Oktay showed that the number of oocytes and embryos obtained in a group stimulated using letrozol was similar to that of patients stimulated using other protocols [27-31].

\section{Is it safe to simulate patients with cancer?}

Few studies have evaluated the potential risks of stimulating the ovaries in breast cancer patients. Following a protocol of letrozol plus FSH and inducing ovulation with $\mathrm{GnRH}$ agonist ensures low levels of oestrogen, which reduces concern over the safety of COS.

Up to now, differences in survival and recurrence of breast cancer rates have not been observed in patients who underwent COS compared with those who did not. The studies present certain limitations in methodology, such as the insufficient size of the sample group and the short duration of the monitoring [43-45]. One study monitored participants for varying lengths of time (between 272 and 600 days) post COS. No differences were found in the recurrence of breast cancer when compared with those patients who did not undergo COS [31].

Breast cancer patients are usually referred for chemotherapy 6-8 weeks after surgery. However, in the early stages of cancer, the chemotherapy treatment can be delayed until 12 weeks without affecting the prognosis, but the patients need to be referred early for diagnosis by a fertility specialist [46]. It is possible to carry out two cycles of COS in the intervening time between surgery and the start of chemotherapy. This strategy garners the best results in terms of the number of eggs and embryos without demonstrable difference in recurrence rates [43]. Generally, it can be stated that COS does not delay the start of chemotherapy, but the patient needs to be referred early for diagnosis by a fertility specialist. It is also necessary for treatment to preserve fertility to be authorised by the oncologist overseeing the patient's treatment.

Neither the cancerous nor the normal cells in the breast react to the gonadotropins (FSH, LH) nor to hCG [45]. Conversely, there is cellular proliferation and an increase in cancer cell lines ER+ (oestrogen receptor) with exposure to oestrogen, and it is dose-dependent. Exposure to progesterone or clomiphene citrate shows a decline in cellular proliferation in the ER- line, with a mutation of BRCA1. On the other hand, the hCG shows a tendency to protect [48]. These findings support the idea that COS with minimal elevation in levels of estradiol does not present an added risk for breast cancer. 


\section{Conclusion}

Preserving fertility is an option for many women who suffer from breast cancer. Freezing embryos and vitrification of oocytes are the two most common options for treatment and both require previous COS. The COS does not represent a risk for breast cancer. The stimulation protocols using anti-oestrogen agents have proven effective, with a minimal increase in estradiol levels. There are strategies to induce baseline ovarian conditions using $\mathrm{GnRH}$ antagonists, avoiding a delay in starting ovarian stimulation. Ovarian reserves and response are lower in women with breast cancer. Well-designed studies are required to evaluate the long-term safety, survival rates, and remission period after ovarian stimulation in women with breast cancer.

\section{List of abbreviations}

$\begin{array}{ll}\text { IVF } & \text { In Vitro Fertilisation } \\ \text { hCG } & \text { Human Chronic Gonadotropin } \\ \text { FSH } & \text { Follicle Stimulating Hormone } \\ \text { GnRH } & \text { Gonadotrophin Releasing Hormone } \\ \text { LH } & \text { Luteinizing Hormone } \\ \text { COS } & \text { Controlled Ovarian Stimulation } \\ \text { ER } & \text { Oestrogen Receptors } \\ \text { PR } & \text { Progesterone Receptors } \\ \text { AFC } & \text { Antral Follicle Count } \\ \text { FAC } & \text { Fluorouracil Adriamycin (Doxorubicin) Cyclophosphamide } \\ \text { FEC } & \text { Fluorouracil Epirubicin Cyclophosphamide } \\ \text { CMF } & \text { Cyclophosphamide Methotrexate Fluorouracil } \\ \text { AC } & \text { Adriamycin (Doxorubicin) Cyclophosphamide } \\ \text { TAC } & \text { Taxotere (Docetaxel) Adriamycin (Doxorubicin) Cyclophosphamide } \\ \text { TCH } & \text { Taxotere (Docetaxel) Carboplatin Herceptin (trastuzumab) }\end{array}$

\section{Conflicts of interest}

No conflict of interest.

\section{References}

1. GLOBOCAN (2012) Estimated cancer Incidence, Mortality and Prevalence WorldWide in 2012. International Agency for Research on Cancer. World Health Organization [http://globocan.iarc.fr/Default.aspx]

2. Siegel R, Naishadaham D and Jemal A (2013) Cancer Statistics, 2013 Cancer J Clin 63 11-30 DOI: 10.3322/caac.21166

3. Glass AG et al (2007) Breast cancer incidence, 1980-2006: combined roles of menopausal hormone therapy, screening mammography, and estrogen receptor status J Natl Cancer Inst 99(15) 1152-61 DOI: 10.1093/jnci/djm059 PMID: 17652280

4. Martos MC et al (2009) Accurately estimating breast cancer survival in Spain: cross-matching local cancer registers with the National Death Index Rev Panam Salud Pública 26 51-4 DOI: 10.1590/S1020-49892009000700008

5. Hilmestein-Braw R, Peters $\mathrm{H}$ and Faber M (1978) Morphological study of the ovaries of leukemia children Br J Cancer 38 82-7 DOI: $\underline{10.1038 / b j c .1978 .166}$ 
6. Chapman RM, Sutcliffe SB and Malpas JS (1979) Cytotoxic-induced ovarian failure in women with Hodking's disease. I Hormone function JAMA 242 1877-81 DOI: 10.1001/jama.1979.03300170023020 PMID: 480620

7. Blumenfeld $Z$ and Eckman A (2005) Tratamientos oncológicos y supervivencia folicular Adalia Farma 11 33-43

8. Letourneau JM et al (2012) Acute ovarian failure underestimates age-specific reproductive impairment for young women undergoing chemotherapy for cancer Cancer 118 1933-39 DOI: $10.1002 / \mathrm{cncr} .26403$

9. EBCTCG (2005) Effects of chemotherapy and hormonal therapy for early breast cancer on recurrence and 15-year survival: an overview of the randomized trials Lancet $3651687-717$ DOI: 10.1016/S0140-6736(05)66544-0

10. EBCTCG (2012) Comparisons between different polychemotherapy regimens for early breast cancer: meta-analyses of long term outcome among 100,000 women in 123 randomized trials Lancet 379 432-44 DOI: 10.1016/S0140-6736(11)61625-5

11. Lee SJ et al (2006) American Society of Clinical Oncology recommendations on fertility preservation in cancer patients $J$ Clin Oncol 24 2917-31 DOI: 10.1200/JCO.2006.06.5888 PMID: 16651642

12. Kim SS, Klemp J and Fabian C (2011) Breast cancer and fertility preservation Fertil Steril $951535-43$ DOI: 10.1016/j.fertnstert. 2011.01.003 PMID: 21272867 PMCID: $\underline{3939612}$

13. Goldhirsch A et al (2009) Personalizing the treatment of women with early breast cancer: highlights of the St Gallen International Expert Consensus on the Primary Therapy of Early Breast Cancer 2013 Ann Oncol 19-29

14. De Laurentiis M, Cancello G, D’Agostino D, Giuliano M, Giordano A and Montagna E et al (2008) Taxane-based combinations as adjuvant chemotherapy of early breast cancer: a meta-analysis of randomized trials J Clin Oncol 26 44-53

15. Tham YL et al (2007) The rates of chemotherapy-Induced amenorrhea in patients treated with adjuvant doxorubicin and cyclophosphamide followed by a taxane Americal journal of clinical Oncology 30 126-132 DOI: 10.1097/01.coc.0000251398.57630.4f

16. Sergentanis TN, Diamantaras AA, Perlepe C, Kanavidis P, Skalkidou A and Petridou ET (2014) IVF and breast cancer: a systematic review and meta-analysis Hum Reprod Update 20 106-23

17. Siristatidis $C$ et al (2013) Controlled ovarian hyperstimulation for IVF: impact on ovarian, endometrial and cervical cancer a systematic review and meta-analysis Hum Reprod Update 19 105-23 DOI: 10.1093/humupd/dms051

18. van Leeuwen FE et al (2011) Risk of borderline and invasive ovarian tumors after ovarian stimulation for in vitro fertilization in a large Dutch cohort Hum Reprod 26 3456-65 DOI: 10.1093/humrep/der322 PMID: 22031719 PMCID: 3212878

19. Doyle P et al (2002) Cancer incidence following treatment for infertility at a clinic in the UK Hum Reprod 17(8) 2209-13 DOI: 10.1093/humrep/17.8.2209 PMID: 12151460

20. Brinton LA et al (2004) Breast cancer risk associated with ovulation-stimulating drugs Hum Reprod 19 2005-13 DOI: $10.1093 /$ humrep/deh371 PMID: 15217997

21. Sönmezer $M$ et al (2010) Breast cancer diagnosis following ovarian stimulation: Are the tumors different? Reprod Biomed Online 21 266-71 DOI: 10.1016/j.rbmo.2010.05.006

22. Wallace WH, Kelsey WT and Anderson RA (2012) Ovarian cryopreservation: experimental or established and a cure for the menopause? Reprod Biomed Online 25 93-95 DOI: 10.1016/j.rbmo.2012.06.008 PMID: 22858254

23. Donnez J et al (2011) Children born after autotransplantation of cryopreserved ovarian tissue. a review of 13 live births Ann Med 43 437-50 DOI: $\underline{10.3109 / 07853890.2010 .546807}$ PMID: $\underline{21226660}$

24. Anderson RA, Wallace WHB and Baird T (2008) Ovarian cryopreservation for fertility preservation: indications and outcomes Reproduction 136 681-9 DOI: 10.1530/REP-08-0097 PMID: 18682546 
25. Cobo A et al (2014) Obstetric and perinatal outcome of babies born from vitrified oocytes Fertil Steril 102(4) 1006-15 DOI: 10.1016/j.fertnstert.2014.06.019 PMID: 25064408

26. Sonmezer M and Oktay K(2006) Fertility preservation in young women undergoing breast cancer therapy Oncologist $11422-36$ DOI: 10.1634/theoncologist.11-5-422 PMID: 16720842

27. Oktay $\mathrm{K}$ et al (2006) Letrozole reduces estrogen and gonadotropin exposure in women with breast cancer undergoing ovarian stimulation before chemotherapy J Clin Endocrinol Metab 91 3885-90 DOI: 10.1210/jc.2006-0962 PMID: 16882752

28. Oktay K et al (2010) GnRH agonist trigger for women with breast cancer undergoing fertility preservation by aromatase inhibitor/FSH stimulation Reprod Biomed Online 20 783-8 DOI: 10.1016/j.rbmo.2010.03.004 PMID: 20382080 PMCID: $\underline{3557820}$

29. Oktay K, Buyuk E and Davis O (2003) Fertility preservation in breast cancer patients: IVF and embryo cryopreservation after ovarian stimulation with tamoxifen Hum Reprod 18 90-5 DOI: 10.1093/humrep/deg045 PMID: 12525446

30. Reddy J and Oktay $\mathrm{K}(2012)$ Ovarian stimulation and fertility preservation with the use of aromatase inhibitors in women with breast cancer Fertil Steril 98 1363-9 DOI: 10.1016/j.fertnstert.2012.09.022 PMID: 23058686

31. Oktay K (2005) Further evidence on the safety and success of ovarian stimulation with letrozole and tamoxifen in breast cancer patients undergoing in vitro fertilization to cryopreserve their embryos for fertility preservation J Clin Oncol $233858-9$ DOI: $10.1200 / J C O .2005 .04 .011$ PMID: 15911867

32. Checa M et al (2012) The effects of letrozole on ovarian stimulation for fertility preservation in cancer- affected women Reprod Biomed Online 24 606-10 DOI: 10.1016/j.rbmo.2012.02.020

33. Tulandi T, Martin J, Al-Fadhli R, Kabli N, Forman R and Hitkari J et al (2006) Congenital malformations among 911 newborns conceived after infertility treatment with letrozole or clomiphene citrate Fertil Steril 85 1761-5

34. Quintero B et al (2010) Ovarian stimulation for fertility preservation in patients with cancer Fertil Steril 93 865-7 DOI: $10.1016 /$. fertnstert.2008.10.007

35. Von Wolff M et al (2009) Ovarian stimulation to cryopreserve fertilized oocytes in cancer patients can be started in the luteal phase Fertil Steril 92 1360-5 DOI: 10.1016/j.fertnstert.2008.08.011

36. Speroff L and Fritz M (2005) Clinical Gynecologic Endocrinology and Infertility (Philadelphia, PA: Lippincott Williams and Wilkins)

37. Domingo $\mathrm{J}$ et al (2012) Ovarian response to controlled ovarian hyperstimulation in cancer patients is diminished even before oncological treatment Fertil Steril 97 930-4 DOI: 10.1016/j.fertnstert.2012.01.093 PMID: 22283969

38. Friedler S et al (2012) Ovarian response to stimulation for fertility preservation in women with malignant disease: a systematic review and meta-analysis Fertil Steril 97125-33

39. Anderson R and Wallace W (2013) Antimüllerian hormone, the assessment of the ovarian reserve, and the reproductive outcome of the young patient with cáncer Fertil Steril 99(6) 1469-75 DOI: 10.1016/j.fertnstert.2013.03.014 PMID: 23541317

40. Ebbel E et al (2011) Reproductive aged women with cancer have a lower antral follicle count than expected Fertil Steril 96 S199-S200

41. Oktay K et al (2010) Association of BRCA1 mutations with occult primary Ovarian insufficiency possible explanation for the link between infertility and breast ovarian cancer J Clin Oncol 28 240-4 DOI: 10.1200/JC0.2009.24.2057

42. Dahhan T et al (2013) Tamoxifen or letrozole versus standard methods for women with estrogen-receptor positive breast cancer undergoing oocyte or embryo cryopreservation in assisted reproduction Cochrane Database Syst Rev 11 Art No.: CD010240 DOI: 10.1002/14651858.CD010240.pub2 PMID: 24213953 
43. Yager JD and Davidson N (2006) Estrogen carcinogenesis in Breast Cancer N Engl J Med 354 270-82 DOI: 10.1056/NEJMra050776 PMID: 16421368

44. Oktay K et al (2005) Fertility preservation in breast cancer patients: a prospective controlled comparison of ovarian stimulation with tamoxifen and letrozole for embryo cryopreservation J Clin Oncol 23 4347-53 DOI: 10.1200/JC0.2005.05.037 PMID: $\underline{15824416}$

45. Turan $\vee$ et al (2013) Safety and feasibility of performing two consecutive ovarian stimulation cycles with the use of letrozole-gonadotropin protocol for fertility preservation in breast cancer patients Fertil Steril 100 1681-5 DOI: 10.1016/j. fertnstert.2013.08.030 PMID: 24055050 PMCID: 3888552

46. Lohrisch C et al (2006) Impact on survival of time from definitive surgery to initiation of adjuvant chemotherapy for early-stage breast cancer J Clin Oncol 24 4888-94 DOI: 10.1200/JCO.2005.01.6089 PMID: 17015884

47. Boukadi S et al (2012) Impact of infertility regimens on breast cancer cell: follicle-stimulating hormone and luteinizing hormone lack a direct effect on breast cell proliferation in vitro Fertil Steril 97 440-4 DOI: 10.1016/j.fertnstert.2011.11.020

48. Cooley A (2012) Effect of infertility and pregnancy-related hormones on breast cell proliferation in vitro Human Reprod 27 146-52 DOI: $\underline{10.1093 / \text { humrep/der378 }}$ 